Revista de la red interuniversitaria de estudios sobre las literaturas rioplatenses contemporáneas en Francia

Nuevas experiencias editoriales y literaturas contemporáneas

\title{
Micropolíticas de la edición y de la traducción : el caso de Colección Chapita
}

\section{Santiago Venturini}

\section{OpenEdition}

\section{Journals}

Edición electrónica

URL: http://journals.openedition.org/lirico/2148

DOI: $10.4000 /$ lirico.2148

ISSN: 2262-8339

Editor

Réseau interuniversitaire d'étude des littératures contemporaines du Río de la Plata

Referencia electrónica

Santiago Venturini, « Micropolíticas de la edición y de la traducción : el caso de Colección Chapita », Cuadernos LIRICO [En línea], 13 | 2015, Puesto en línea el 15 diciembre 2015, consultado el 30 abril 2019. URL : http://journals.openedition.org/lirico/2148; DOI : 10.4000/lirico.2148

Este documento fue generado automáticamente el 30 abril 2019.

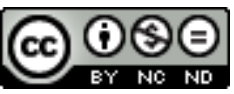

Cuadernos LIRICO está distribuido bajo una Licencia Creative Commons Atribución-NoComercialSinDerivar 4.0 Internacional. 


\title{
Micropolíticas de la edición y de la traducción : el caso de Colección Chapita
}

\author{
Santiago Venturini
}

1 A fines de los 90, la crisis de la industria editorial argentina alcanzaba un punto álgido, cuyos signos más visibles eran la marcada polarización del campo editorial y la concentración de la actividad en manos de grupos transnacionales (Botto 212). Al mismo tiempo, a lo largo de ese período y en el marco de un proceso de "convergencia tecnológica" (Lerman 2013), se producía una apropiación generalizada de las nuevas tecnologías, las cuales tuvieron, entre otros efectos, un impacto importante en la cadena de producción del libro. En un trabajo publicado en 2001 -aunque ya se habían llevado a cabo observaciones similares anteriormente (Harari 2000)-, Celina Manzoni señalaba la trascendencia de la cuestión tecnológica para las pequeñas editoriales literarias :

Como resultado de los avances en el software tanto para edición como para comercialización, se han abaratado los costos, se han diversificado las posibilidades y resulta posible editar muchos más títulos que hace diez años, también ha mejorado mucho la tecnología gráfica disminuyendo los costos de imprenta (Manzoni 790).

2 Efectivamente, la cuestión tecnológica resulta imprescindible para comprender el surgimiento de nuevas formas de pensar y practicar la edición, más o menos profesionalizadas, que fueron agrupadas bajo el rótulo amplio de "editoriales independientes" (Sarachu 2013 ; Balaguer 2014). La adopción de estas nuevas tecnologías modificó considerablemente la cartografía editorial, y continúa haciéndolo : en una mesa de discusión reciente, Ana Ojeda, escritora y editora de El octavo loco, señalaba que actualmente "hay la misma cantidad de editoriales que de escritores y hasta que de lectores, porque técnicamente se ha facilitado muchísimo el hecho de hacer un libro" (Ojeda, Ríos y Almada 2014).

3 En esta escena abierta ya desde fines de los 90, y luego de la consolidación de formaciones que pueden considerarse relevantes para su surgimiento -como el de las editoriales cartoneras- aparece en 2009 Colección Chapita. Se trata de una editorial artesanal de 
poesía dirigida por los poetas Daniel Durand -responsable de "Ediciones Deldiego" entre 1998 y 2001- y Matías Heer ${ }^{1}$. Basta una inspección rápida de los títulos publicados accesibles hoy, casi en su totalidad, a través de la web ${ }^{2}$ - para advertir la naturaleza de la propuesta. Colección Chapita hace de la edición una práctica de carácter doméstico: publica libros manufacturados de tirada reducida, con portadas serigrafiadas y detalles únicos, irrepetibles. En una entrevista, Daniel Durand describe el proceso de confección de cada título :

Lo hacemos todo en mi casa. Primero voy, compro el cartón, lo hago cortar, después lo doblo, lo pegamos, si tenemos el texto diseñamos los interiores en InDesign, PageMaker o Quark Press, imprimimos los interiores y los pegamos a la tapa. A la tapa, antes de pegarla al interior, la serigrafiamos : primero el fondo, después la tipografía, y después lo que nosotros llamamos el 'tunning', que son las cosas que le ponemos arriba de las tapas, como las chapitas o las cosas que vamos inventando. Un día se nos ocurrió la chapita, que calzó perfecto, salió de una manera fácil y fue lo que dio el nombre y queda muy bien. Es todo artesanal, cada libro es único... (Durand y Heer 2009).

Este proceso de fabricación está ligado a una ideología de la edición que pone en el centro cierta idea de libertad -la misma que Durand destaca con respecto a su propia escritura ${ }^{3}$ y que aparece como la contrapartida del proceso de producción industrial y convencional del libro. Por esa razón, puede afirmarse que el procedimiento casero que da forma a los títulos de Colección Chapita es, al mismo tiempo, una forma alternativa de fabricar un libro, una posición crítica y un acto de resistencia ${ }^{4}$. La editorial fabrica objetos que son libros, objetos no seriados, objetos de excepción a los que la intervención artística sobre el emplazamiento más externo, la portada, les asigna, a través del detalle irrepetible -las chapitas- una marca de individualidad que anula la posibilidad de la copia y los diferencia de los otros libros. Hay en esa voluntad de producir objetos únicos una relación espontánea y obvia con las afirmaciones que Walter Benjamin hace en las primeras páginas de La obra de arte en la era de su reproducción técnica, afirmaciones, paradójicamente, reproducidas hasta el cansancio : la técnica implica una "devaluación del hic et nunc" de la obra de arte, un atentado contra su autenticidad, una destrucción de su aura, dado que "reemplaza el lugar de la existencia irrepetible por la repetición masiva" (Benjamin 2011 11-12). Pero aparece también un vínculo con aquello que Benjamin afirma al comienzo de una obra anterior, Calle de mano única :

La eficacia literaria trascendente solo puede consumarse en la estricta alternancia entre hacer y escribir ; debe cultivar los discretos formatos del volante, el folleto, el artículo de revista y el cartel publicitario, que se corresponden mejor con su influencia en las comunidades activas que el pretencioso gesto universal del libro. Sólo este lenguaje inmediato se muestra eficazmente a la altura de la época (Benjamin 2014 43).

Claro que Benjamin está hablando del "lenguaje inmediato" de la modernidad, pero lo que subsiste en Colección Chapita es la idea de que también la edición necesita volverse más inmediata, modificarse como práctica para estar "a la altura de la época" y escapar al "gesto universal del libro" a través de la confección de libros, sí, pero libros que reformulan el soporte mismo. Al igual que otros sellos surgidos durante los 90, Colección Chapita produce libros en los márgenes de la industria y el mercado editoriales, por eso se trata no de una editorial sino de una "editorialcita" (Durand 2010). Esos libros, producto de un trabajo manual, exponen la manufactura y hacen de ella un valor, al igual que la artesanía. Por eso mismo escapan a lo institucionalizado, escapan al control editorial y la descripción bibliográfica: no cuentan con una página de legales, no consignan datos 
editoriales, no tienen ISBN (aunque sí cuentan con un colofón, que aporta una información mínima sobre el volumen). Esta resistencia a lo institucionalizado, a lo normalizado, resulta central no sólo porque significa, para sellos como Colección Chapita, una libertad innegable -la de publicar lo que quieren, la de traducir lo que quieren y como quieren, sin someterse a la maquinaria legal que regula la actividad editorial (derechos de traducción, contratos) y sin seguir los protocolos adoptados por los grandes sellos en la publicación de cada título- sino y sobre todo porque habla de su concepción de la edición como un acto de intervención : la desprofesionalización -debería revisarse el alcance del término "profesionalización", que utilizo ahora en un sentido netamente empresarial- y la desindustrialización hacen de la edición una nueva forma de "activismo cultural" (Vanoli 176).

6 La idiosincrasia de Colección Chapita se construye, por un lado, en la producción artesanal de sus libros (debería pensarse la relación entre el énfasis en la materialidad del libro y las escrituras que Colección Chapita publica: las escrituras no pueden leerse independientemente del soporte que las pone en circulación); por otro lado, esa idiosincrasia se sostiene en decisiones de catálogo : la inclusión de poetas locales, más bien jóvenes (algunos del interior del país, como Fernando Callero, Matías Heer, Julián Bejarano, Ariel Delgado y Antolín, entre otros), pero también la inclusión de autores extranjeros nunca antes traducidos o escasamente traducidos en nuestro país como Craig Raine o John Berryman. Estas dos operaciones (la exacerbación de la dimensión material del libro y la conformación de un catálogo) dan cuenta de una micropolítica de la edición -y, como veremos, de la traducción-, la cual puede definirse, en términos básicos, como "la creación de espacios transversales en el medio de los espacios que distribuyen e imponen las políticas mayores" (Astutti y Contreras 773).

\section{Dos nociones de traducción}

7 En un artículo reciente, Damián Tabarovsky señaló la trascendencia que las editoriales surgidas en nuestro país durante la última década -e incluso antes-, tienen como importadoras de autores extranjeros; tanta, que "si las editoriales pequeñas argentinas no existieran, prácticamente no habría traducciones hechas en Argentina" (2014). Es innegable que existe un conjunto de editoriales ${ }^{5}$ que hicieron y hacen de la traducción una práctica crucial no sólo para dar forma a sus catálogos sino también como recurso para delinear un perfil editorial propio.

Colección Chapita es una editorial traductora : las traducciones constituyen casi la mitad de los títulos que aparecen en su catálogo. Más interesante que esta comprobación es otra, inmediata: los títulos traducidos se agrupan en dos colecciones diferentes: "Traducciones" y "Traducciones chapita" 7 . La distinción entre ambas colecciones no obedece al corpus de autores seleccionados sino a una diferencia de método, de estrategias traductoras, que tanto la denominación "traducciones chapita" -con esa locución proveniente del lunfardo, cuyo significado no es sólo loco, sino también extravagante, raro, irreflexivo (Conde 2011 459)- como algunos de los títulos -Doce escritos petisos, $j A$ las puteadas! o Cumbias finas- anticipan. A través de la distinción entre ambas colecciones, el catálogo de Colección Chapita expone dos nociones y, por lo tanto, dos modos de practicar la traducción. Por un lado, una noción convencional, a través de la cual se presentan versiones en castellano de un conjunto de autores -en su mayoría norteamericanos : John Ashbery, Wallace Stevens, Elizabeth Bishop, Delmore Schwartz-, 
guiadas por una idea de equivalencia con respecto al texto extranjero. Esta equivalencia se define como el parámetro transversal de la colección "Traducciones", por contraposición a los parámetros que organizan la otra colección. Porque, por otro lado, el concepto de "traducción chapita" - ese tipo de traducción extremadamente experimental, "libertaria" pero también "demoledora" (Durand 2013) - desafía la noción convencional de traducción.

La distancia entre un concepto y otro aparece expuesta en algunos de los paratextos de los títulos de Colección Chapita. La introducción que redacta Matías Heer para la antología Cáncer joven con un plan, de John Berryman, funciona como el manifiesto de la "traducción chapita" y es un marco para la colección :

Las traducciones no tienen por qué ser reproducciones, en todo caso podrían ser interpretaciones y remasterizaciones de los textos. Así, la influencia no debe ser una mochila de animales exóticos que uno trae del extranjero para desempacarla en un hábitat diferente donde el clima y demás factores debilitan al tucán malayo, sino una fábrica de acontecimientos que mutan al texto y lo incorporan dentro de la tradición rioplatense (...) La traducción, como una operación de conexiones, funciona a partir de la remodelación, naturalizando y execrando la otra lengua, para finalmente traducir dentro de la propia cultura en un español libre, argótico, moderno, rioplatense y bien chapita (Berryman 2008)

¿Cómo se define la traducción chapita en términos de estrategias? Se trata de traducciones guiadas "más por los significantes que por los significados", que traspasan "sonidos e interpretaciones dislocadas" según "un capricho delirante" (Berryman 8). En palabras de Durand, son traducciones basadas en "juegos completamente creativos" cuyo fin es "faltarle el respecto al texto original todo el tiempo" (Durand 2013). O como lo señala Matías Heer en el prólogo a Cumbias finas de Guilhem Peitieus (su versión de Guillermo de Poitiers): "en estas versiones me propuse hacer uso nuevamente de la ecuación etimológica : traducción, tradición, traición" (Peitieus). Es decir, el concepto de "traducción chapita" replica en otra instancia la idea de libertad y de idiosincrasia que la editorial se plantea en la confección de sus libros.

11 Daniel Durand traduce en 1988 el poema "El cuervo" de Edgard Allan Poe, publicado en Colección Chapita dos décadas después. Este ejercicio es el claro antecedente de la "traducción chapita". La irreverencia es insuperable, porque Durand desconoce la lengua en la que Poe escribió su poema, ya que casi no sabe inglés :

una de las primeras cosas que hice con la traducción, antes de saber ninguna lengua extranjera fue traducir entero el cuervo de Poe, sin saber ni una palabra de inglés, y traducía a lo que me sonaba en español, quedó un texto muy libre e inconexo, pero muy divertido fue hacerlo. El texto en inglés como sabemos se llama 'The Raven' y mi traducción se llamaba "La rabia" (2006).

12 La transposición del título da el tono de todo lo que se leerá. La estrategia de traducción es la homofonía: el sonido de las palabras en inglés evoca palabras en castellano acústicamente similares, pero la similitud fonética implica un desvío completo del sentido. En este caso, la traducción se publica en formato bilingüe, con el texto en inglés y el texto en castellano en páginas enfrentadas, para que el lector pueda advertir la estrategia (esto no es una constante: ciertos títulos de la colección colocan el texto extranjero en las últimas páginas, mientras que otros no lo incluyen). Algunos ejemplos : "bleak December" (sombrío diciembre) se vuelve "el bleque del invierno", "the floor" (el suelo) se vuelve "las flores", el adverbio "merely" (simplemente) es "merengue", "lonely" (solitario) es "leones", "tapping" (golpeteo) es "tapia". No se trata de una estrategia uniformemente aplicada a lo largo del texto : en ocasiones algunos vocablos se traducen 
de manera adecuada y en otras se insertan reformulaciones y agregados que no tienen ninguna relación con el texto en inglés pero resultan necesarios para completar el sentido del nuevo verso en castellano. En esta estrategia se tiende, además -esta sí es una constante en las traducciones chapita- a la elección, en castellano, del término más localizado, el más vernáculo, como es el caso de "bleque del invierno", que recupera un vocablo lunfardo que proviene de una probable deformación del vocablo inglés "black" (Conde 2004 33) y cuyo significado es "alquitrán".

13 El resultado de la estrategia de homofonía es una segmentación de "El cuervo" en secuencias inconexas que se leen como poemas disparatados. Así, los versos "But the raven, sitting lonely on the placid bust, spoke only/That one Word, as if soul in that one Word he did outpour" (Poe 2006 424) ${ }^{8}$ se transforman en: "Pero la rabia carcomida de los leones sobre las plácidas tetas/es poco solamente./ Que una palabra como el sonido del mundo estaba pobre y afuera" (Poe La rabia). La eficacia de la estrategia se mide sólo en la extensión de un verso o de un fragmento de verso, cuando consigue dar una imagen nueva, inesperada y crea algo nuevo a partir del poema de Poe. El mismo Durand lo señala : es una estrategia que "funciona en partes, nada más" (2013).

Como estrategia, la homofonía se relaciona con aquello que André Lefevere ha denominado "traducción fonética" (1975 22), práctica que intenta reproducir en la lengua meta la configuración sonora del texto fuente (Lefevere toma como ejemplo las versiones de Catulo en inglés, firmadas por Celia y Louis Zukofsky) ${ }^{9}$. Aunque la traducción fonética fuerza y manipula la lengua de llegada para alcanzar su objetivo, nunca logra traducir el sonido de la obra extranjera ya que, como lo señala Lefevere, "el texto meta es simplemente una aproximación a los sonidos del texto fuente filtrados a través de la 'grilla fonética' de esa lengua" (23). No obstante, en el caso de las traducciones chapita el fin de la homofonía no es reproducir los sonidos de otra lengua a través de una experimentación con los límites del castellano, sino partir de esa otra configuración sonora para dar lugar a un nuevo texto que no pretende funcionar como la traducción de un "original", sino que se distancia de él. La homofonía es, básicamente, un mecanismo productor de textualidad. La estrategia recuerda, más bien, al procedimiento que estuvo en la base de las obras de Raymond Roussel, y que el mismo Roussel expuso de manera minuciosa en una obra póstuma: Cómo escribí algunos libros míos. Este procedimiento consistía en la construcción de frases a través de homonimias y del contraste entre las diferentes acepciones de los homónimos puestos en relación. Cómo escribí algunos libros míos es un detallado catálogo de un procedimiento que adquiere, cada vez, mayor complejidad. Un ejemplo : a partir de dos parónimos, billard (billar) y pillard (saqueador, bandido), Roussel construye dos oraciones que, utilizando los mismos términos, tienen sentidos completamente diferentes: Les lettres du blanc sur les bandes du vieux billard... (donde lettres tiene la acepción de "letras", blanc la de "tiza" y bandes la de "orlas") y Les lettres du blanc sur les bandes du vieux pillard... (donde lettres significa "cartas", blanc "hombre de raza blanca" y bandes "hordas guerreras"). El objetivo, afirma Roussel, era escribir un cuento que comenzara con la primera oración y concluyera con la segunda (Roussel 25), es decir, hacer de ese trabajo con la semejanza acústica y el contraste semántico un procedimiento compositivo. Como lo señala algunas páginas más adelante, "este procedimiento evolucionó y me llevó a tomar una frase cualquiera, de la que extraía imágenes, dislocándola al modo de un jeroglífico" (36). La operación no tiene límites : "Me servía de cualquier material (...) Llegué incluso a utilizar el nombre y las señas de mi zapatero : 'Hellstern, 5, Place Vendôme', que convertí en Hélice tourne zinc plat se rend dôme 
(Hélice gira aeroplano se convierte -se rendre equivalía aquí a convertirse- en cúpula)" (38). Este último ejemplo es importante porque pone en evidencia la misma lógica que está presente en la estrategia de la homofonía de las traducciones chapita : no traducir, sino construir a partir de cierta configuración sonora -la del texto extranjero- otra similar en una nueva lengua en la que sin embargo el sentido acaba siendo borrado por el juego acústico.

que sucede con Douze pétits écrits (Doce pequeños escritos) de Francis Ponge, que en la traducción de Durand y Heer se vuelve Doce escritos petisos. La estrategia es la misma: "maintenant" (ahora) es "mantengo", "façon" (manera) es "facón", "tête" (cabeza) es "teta", "paraîtra" (parecerá) es "para atrás". El sintagma : "Ma tête de mort paraitra dupe de son expression" ("Mi calavera parecerá víctima de su expresión") se torna : "Mi teta de muerto para atrás, dopada de su expresión".

La homofonía y las adiciones o agregados son los procedimientos primordiales. Es cierto que cada traducción chapita ejerce un control diferente de estas estrategias. En algunas de ellas, su aplicación no controlada lleva al absurdo (que implica solo una variación de grado en relación con otras). Es el caso de Las flores de más, de Carlos Baudelongher -un híbrido entre Charles Baudelaire y Néstor Perlongher- que traducen Durand, Heer y Fadel. Las flores de más se inicia con una versión -este término ya no es válido- del primer poema de Las flores del mal de Charles Baudelaire, "Au lecteur" ("Al lector"). Los primeros versos del poema de Baudelaire, en francés, dicen :

La sottise, l'erreur, le péché, la lésine,

Occupent nos esprits et travaillent nos corps,

Et nous alimentons nos aimables remords,

Comme les mendiants nourrissent leur vermine.

En la traducción de Las flores del mal de Américo Cristófalo, la última traducción íntegra del libro publicada en Argentina (2006), esos versos aparecen así :

La estupidez, el error, el pecado, la avaricia,

ocupan nuestro espíritu, nos trabajan el cuerpo,

$\mathrm{y}$ alimentamos amables remordimientos,

como los mendigos dan de comer a sus larvas.

Finalmente, en los primeros versos de "Al lactear" de Las flores de más se lee :

A la petisa, le erré, la peché, la lesioné

Ocupan unos espíritus travestis nuestros cuerpos

Y nuestros alimentos los animales muerden

Como las medialunas que nos arrasaron los vermines.

Como es posible advertir, las traducciones chapita se basan en una denegación del concepto mismo de traducción, hacen del texto extranjero una excusa, una traza sobre la que se acaba construyendo otra cosa, algo que tiene finalmente algunas marcas extremadamente mínimas de semejanza con ese texto inicial : no los títulos de libros o poemas, ni siquiera, a veces, el nombre que firma ese texto -como lo demuestran Guilhem Peitieus o Carlos Baudelongher- sino una especie de murmullo, un eco sonoro.

La lectura de estas traducciones podría detenerse ahí : en la percepción de la irreverencia, del gesto descabellado. Y es necesario dejar en claro que se trata, en gran parte, de eso : la confección de libros mínimos -algunos de 15 páginas- que habilitan un juego con los textos que no tiene otro sentido más que el juego en sí mismo. No obstante, es válido preguntarse si la noción y la práctica de la "traducción chapita" y su énfasis en la pura experimentación tienen un alcance mayor. Durand da una respuesta indolente: 
"Realmente no sé si esa cosa tan lúdica tendrá cierto valor literario, más allá del experimento" (2013). Creo que es necesario leer la noción en diferentes niveles. Es evidente que la "traducción chapita" es una operación estética y cultural en la que se somete al texto extranjero al poder absorbente de una lengua híper localizada, cuyo efecto es la aclimatación extrema y el borramiento de la extrañeza de ese texto. Es indudable que la estrategia resulta interesante, sobre todo cuando se trata de autores considerados clásicos y numerosas veces traducidos -como Edgard Allan Poe o Charles Baudelaire-, dado que supone un acercamiento novedoso a textos que parecen estar petrificados en el lugar canónico que ocupan.

Pero al mismo tiempo, es necesaria una lectura contextual de la noción de traducción chapita, noción que está vinculada con la naturaleza de Colección Chapita como proyecto editorial y con su posicionamiento marginal en el panorama de la edición. Porque el concepto de "traducción chapita", como lo señalé antes, traslada a la práctica de la traducción la misma voluntad de experimentación, la misma exacerbación de la dimensión material y de la manufactura que la editorial pone en juego en la fabricación del objeto libro. En primer lugar, porque las traducciones chapita, al igual que los ejemplares que fabrica la editorial, son únicas, irrepetibles, están hechas de excepción: son lo que no son las otras traducciones. En segundo lugar, porque la "traducción chapita", en un movimiento que es estético pero también político -o micropolítico-, reniega del ejercicio "serio" e institucional de la práctica y se postula como un ejercicio menor, un divertimento. Las traducciones chapita son traducciones manufacturadas, caseras y por eso mismo irreverentes, pero también parciales, incompletas, desprolijas, malas. En este sentido, la traducción chapita es un experimento que adquiere valor solo en el marco del proyecto editorial que la incluye : más allá de ese marco, la noción pierde alcance y sucumbe al juicio admonitorio que cualquier lectura demasiado responsable se apresura a proferir. Los libros de Colección Chapita no presentan a Poe, Baudelaire o Berryman mal traducidos : o sí, tal vez lo hacen, precisamente para mostrar que Poe, Baudelaire y Berryman pueden ser secuestrados por una formación editorial que reniega tanto de la fabricación, la circulación y el peso oficial del libro como del ejercicio institucionalizado de la traducción.

\section{BIBLIOGRAFÍA}

\section{Bibliografía}

Fuente

"Colección Chapita" : http://coleccionchapita.tumblr.com/

Baudelaire, Charles. Las flores del mal. Américo Cristófalo, traductor. Buenos Aires : Colihue, 2006.

Berryman, John. Cáncer joven con un plan. Daniel Durand y Matías Heer, traductores. Buenos Aires : Colección Chapita.

Peitieus, Guilhem. Cumbias finas. Matías Heer, traductor. Buenos Aires : Colección Chapita. 
Poe, Edgard Allan. La rabia. Daniel Durand, traductor. Buenos Aires : Colección Chapita.

Poe, Edgard Allan. The Portable Edgard Allan Poe. London: Penguin Books, 2006.

Poe, Edgard Allan. Poemas. Sevilla : Publicaciones de Aula de Letras, 2012.

Teoría, crítica y entrevistas

Astutti, Adriana y Contreras, Sandra. "Editoriales independientes, pequeñas... Micropolíticas culturales en la literatura argentina actual”. Revista Iberoamericana, 197, 2001 : 767-780.

Balaguer, Miguel. “Nuestro libro más vendido es un libro de poesía”. Entrevista de Santiago Venturini, Bazar Americano 47 (2014). Disponible en : http://www.bazaramericano.com/ reportajes.php?cod=31\& pdf $=$ si $(12 / 07 / 2014)$.

Benjamin, Walter. La obra de arte en la era de su reproducción técnica. 1937-1938. Buenos Aires : Cuenco de Plata, 2011.

Benjamin, Walter. Calle de mano única. 1928. Jorge Monteleone, edición y prólogo. Buenos Aires : Cuenco de Plata, 2014.

Botto, Malena. “1990-2000. La concentración y la polarización de la industria editorial”. Editores y políticas editoriales en Argentina, 1880-2000, ed. José Luis De Diego. Buenos Aires : Fondo de Cultura Económica, 2006. 209-249.

Conde, Oscar. Lunfardo. Un estudio sobre el habla popular de los argentinos. Buenos Aires : Taurus, 2011.

Conde, Oscar. Diccionario etimológico del lunfardo. Buenos Aires : Taurus, 2004.

De Campos, Haroldo. "De la traducción como creación y como crítica". 1963. De la razón antropofágica (y otros ensayos). Rodolfo Mata, selección, traducción y prólogo. Siglo XXI : México, 2000. 185-204.

Durand, Daniel. “Entrevista a Daniel Durand". Entrevista de Pedro Mairal. 2006. Disponible en : http://elseniordeabajo.blogspot.com.ar/2006/08/entrevista-daniel-durand.html (13/07/2014).

Durand, Daniel. “Daniel Durand. Cielos, personas, flash”. Entrevista de Damián Ríos y Mariano Blatt. Otra Parte, 8, (2008). Disponible en : http://www.revistaotraparte. com/n \%C2 \%BA-14-oto \% C3 \%B1o-2008/daniel-durand-cielos-personas-flash (10/07/2014).

Durand, Daniel. "Colección Chapita”. Entrevista de Sol Echeverría y Daniela Szpilbarg. Noretornable, 6, (2010). Disponible en : http://www.no-retornable.com.ar/v7/dossier/durand.html (25/08/2014).

Durand, Daniel y Heer, Matías. "Daniel Durand y Matías Heer hablan de Editorial Chapita". Revista Proa, 2009. Disponible en : http://proa.org/esp/news/2009/ 03/daniel-durand-y-matiasherr-hablan-de-editorial-chapita/ [10/05/2013] (13/06/2014).

Durand, Daniel. “Bardo, chispazo. Conversación con Daniel Durand”. Alba. Lateinamerika Lesen, 2, (2013). Disponible en : http://www.albamagazin.de/es/mediadores/ 70-bardo-chispazo.html (10/07/2014).

Harari, Pablo. “Existe hoy en día una amenaza sobre la edición independiente ?”. Primer Encuentro de Editores Independientes de América Latina, 2000. Disponible en : http://www.oei.es/ cultura2/actas02.htm [13/06/2014].

Lefevere, André. Translating Poetry, Seven strategies and a Blueprint. Amsterdam : Van Gorcum, 1975. 
Lerman, Gabriel. “Convergencia tecnológica, pueblo y nación”. La grieta. Política, economía y cultura después de 2001. Sebastián Pereyra, Gabriel Vommaro y Germán Pérez, editores. Buenos Aires : Biblos, 2013. 223-235.

Manzoni, Celina. "¿Editoriales pequeñas o pequeñas editoriales ?", Revista Iberoamericana, 197 (2001) : 781-793.

Ojeda, Ana ; Ríos, Damián y Almada, Marcos. "Publicamos lo que nos conmueve”, 2014. Disponible en : http://blog.eternacadencia.com.ar/archives/2014/35695 [17/10/2014].

Roussel, Raymond. Cómo escribí algunos libros míos. Trad. de Pedro Gimferrer. Barcelona : Tusquets, 1973.

Sarachu, Julia. "Mis traducciones son parte de mi obra". Entrevista de Santiago Venturini. Bazar Americano, 43, 2013. Disponible en : http://www.bazarame ricano.com/reportajes.php ?cod $=27 \&$ $\mathrm{pdf}=\mathrm{si}(27 / 08 / 2013)$.

Tabarovsky, Damián. "Literatura argentina reciente : cuanto más marginal, más central”. Letras libres, noviembre de 2014. Disponible en : http://www.letraslibres.com/revista/dossier/ literatura-argentina-reciente-cuanto-mas-marginal-mas-central (13/11/2014).

Vanoli, Hernán. "Pequeñas editoriales y transformaciones en la cultura literaria argentina". Apuntes de Investigación del CECYP, 15 (2009) : 161-185.

\section{NOTAS}

1. En la editorial interviene, casi desde los inicios, el poeta Tomás Fadel. Actualmente, y luego de un tiempo de inactividad, Colección Chapita prepara nuevos títulos.

2. En http://coleccionchapita.tumblr.com/ es posible repasar el catálogo casi completo de la editorial y descargar de manera gratuita sus títulos en formato EPUB. Es interesante no perder de vista esta ambivalencia, la del énfasis en la fabricación manual del objeto libro y su posterior transformación en un soporte virtual, disponible en internet. Esta ambivalencia deja ver diferentes cuestiones, dos al menos : por un lado, la inaccesibilidad de los títulos ya publicados por Colección Chapita, debido a una tirada reducida y a su circuito restringido de circulación ; por el otro, la omnipresencia que ostenta desde hace tiempo la web como plataforma para la exposición y la puesta en circulación de productos editoriales (plataforma que editoriales como estas, de poco capital, no pueden desaprovechar).

3. "Siempre me propongo tener absoluta libertad para escribir, hacerlo desde un lugar personal y no tanto desde una editorial, una revista o una estética fija, negociando lo menos posible o nada con el resto" (Durand 2008).

4. Al hablar de los instrumentos que utiliza para la confección de los libros, como una guillotina de fundición o una prensa de hierro forjado, Durand afirma: "me gustaría que todos los escritores pudieran contar con estas herramientas, y ¡chau imprentas, chau industria editorial!" (2008).

5. Una lista incompleta de nombres con trayectorias y alcances muy disímiles podría incluir a Adriana Hidalgo, Bajo la Luna, Alción, La Bestia Equilátera, Mardulce, Corregidor, El cuenco de plata, Gog y Magog, Eterna Cadencia, Barba de Abejas, Añosluz, Dakota, Luz Mala, entre otras.

6. Los títulos de la colección "Traducciones" son: Autorretrato en un espejo convexo, John Ashbery (trad. de Matías Heer) ; El elemento irracional en la poesía, de Wallace Stevens (no consigna traductor) ; Quince proposiciones falsas contra Dios, de Jack Spicer (trad. de Francisco Bitar); La cebolla memoria, de Craig Raine (trad. de Daniel Durand y Miguel Ángel Petrecca) ; La luna brilla sobre los huesos blancos, de Tu Fu (trad. de Daniel Durand), Pequeño ejercicio, de Elizabeth Bishop (trad. de 
Laura Crespi) ; una antología de poetas japonesas (trad. de Laura Crespi); Seurat : domingo por la tarde a orillas del Sena, de Delmore Schwartz (trad. de José Kozer).

7. Los títulos incluidos en "Traducciones Chapita" son : 12 escritos petisos, de Francis Ponge (versiones de Daniel Durand y Matías Heer) ; ¡A las puteadas!, de Jules Laforgue (versiones de Matías Heer) ; Cáncer joven con un plan, de John Berryman (versiones de Daniel Durand y Matías Heer) ; Catán, de Li Po (versiones de Pablo Cruz Aguirre) ; Cumbias finas, de Guillaume de Poitiers (versiones de Matías Heer); La rabia, de Edgard Allan Poe (versiones de Daniel Durand) ; Las flores de más, de "Carlos Baudelongher" (versiones de Daniel Durand, Tomás Fadel y Matías Heer) ; Los cantos, de Ezra Pound (versiones de Matías Heer) y Viñas desmoronadas, de Su Tung P’o (versiones de Tomás Fadel).

8. En la traducción de J. Pérez Bonaldo se lee: "Mas el cuervo, fijo, inmóvil, en la grave efigie aquella,/ sólo dijo esa palabra, cual si su alma fuese en ella/ vinculada-ni una pluma sacudía, ni un acento/

se le oía pronunciar..." (Poe 2012 92)

9. Lefevere muestra el modo en que los Zukofsky traducen un verso del poema 64 de Catulo: "tum Thetidis Peleus incensus fertur amore" ("Se dice que Peleo fue entonces encendido por el amor de Thetis") como "T'my Thetis this Peleus incandesced fair thru his armor". Una traducción más convencional de ese verso en inglés sería : "Then is Peleus said to have caught fire with love of Thetis..." (Lefevere 23).

\section{RESÚMENES}

Colección Chapita es la editorial artesanal de poesía que dirigen Daniel Durand y Matías Heer desde 2009. Además de la publicación de poetas argentinos contemporáneos, su catálogo cuenta con dos colecciones de traducciones : "Traducciones" y "Traducciones chapita”. Mientras que la primera aparece como una colección "convencional”, la segunda incluye versiones irreverentes, hechas, como lo señalan sus editores, en "un español libre, argótico, moderno, rioplatense y bien chapita". Colección Chapita expone una micropolítica de la traducción que debe ser leída en relación con la posición excéntrica que el sello elige ocupar en el panorama de la edición local.

Colección Chapita, la maison d'édition dirigée par Daniel Durand et Matías Heer depuis 2009, est consacrée à la publication artisanale de poésie. Outre la publication de poètes argentins contemporains, son catalogue inclut deux collections de traductions: «Traducciones» et «Traducciones chapita». Alors que la première se présente comme une collection conventionnelle de traductions, la deuxième propose des traductions faites, comme le signalent les éditeurs, dans « un espagnol libre, argotique, du Rio de La Plata, et assez chapita ». Colección Chapita déploie une micropolitique de la traduction qui pourrait être considérée comme le résultat de la position excentrique que cette maison d'édition occupe dans l'horizon de l'édition argentine.

Colección Chapita, the publishing house run by Daniel Durand and Matías Heer, is dedicated since 2009 to the artisanal publishing of poetry. In addition to the promotion of contemporary Argentinian poets, its catalogue includes two collections of translations: "Traducciones" and "Traducciones chapita". The former is a "conventional" collection of translations, whereas the latter consists of versions made, in the words of the publishers, "in free, modern, slangy, and 
chapita Spanish from the Río de La Plata". Colección Chapita enacts a micropolitics of translation that could be perceived as a particular position adopted in the publishing scene.

ÍNDICE

Mots-clés: traduction, édition indépendante, Colección Chapita

Palabras claves: traducción, editoriales independientes, Colección Chapita

Keywords: translation, independent publishers, Colección Chapita

\section{AUTOR}

SANTIAGO VENTURINI

FHUC, UNL- CONICET 